\title{
Analysis of Air Quality Index obtained via Remote Sensing for Lagos State Urban Area, Nigeria
}

\author{
L.A. Akinyemi ${ }^{1,2}$, A.A. Periola ${ }^{3}$, O.O., Shoewu ${ }^{2}$, \\ A. A. Alonge ${ }^{3}$, K.A Ogudo ${ }^{3}$ \\ ${ }^{1}$ Department of Electrical Engineering, University of Cape Town \\ ${ }^{2}$ Department of Electronic and Computer Engineering, Lagos State University, Lagos, Nigeria \\ ${ }^{3}$ Department of Electrical and Electronics Engineering Technology \\ University of Johannesburg \\ \{1tfaki001@myuct.ac.za, aaperiola@uj.ac.za, engershoewu@yahoo.com, aalonge@uj.ac.za , \\ kingsleyo@uj.ac.za\}
}

Received: $13^{\text {th }}$ June 2017

Accepted: $17^{\text {th }}$ December 2017

Published: $16^{\text {th }}$ March 2018

https://doi.org/10.47545/etrj.2018.3.1.038

\begin{abstract}
Advancements in the field of communications are moving towards the internet of things (IoTs). Wireless Sensor Networks (WSN) can be used to monitor environmental conditions such as pollution using independent sensors communicating with each other using the IoTs technology thereby leading to the design of an accurate environmental monitoring system. Air pollution is a critical environmental challenge which is a major concern in cities and will have to be reduced if cities are to be regarded as smart cities. It has negative short and long term effects on human health that would deter people from moving to cities thereby impairing economic growth. It is important to reduce the effect of air pollution. This can be achieved by using WSN nodes to monitor air pollution. WSN nodes deployed around the city and along traffic routes can be used to achieve this objective. This paper proposes the design of WSN network for monitoring air pollution. In this paper, the stationary sensor nodes are deployed around the city to acquire pollution data. The data acquired by stationary sensor nodes is transmitted to mobile nodes located on vehicles. The received data is processed when mobile nodes reach a pollution monitoring system. These data would be stored locally in its memory with a time and location stamp. Mobile nodes placed on public buses, buildings and vehicles would wirelessly collect the data as they pass by the stationary nodes. The data would then be processed once the nodes arrived back at the pollution monitoring system. In the proposed architecture, the stationary nodes run on ZigBee standard while the mobile nodes run on fifth generation (5G) and Long-time evolution (LTE) technology.
\end{abstract}

Keywords: 5G, Cloud, Pollution, Smart City, WSN

\section{INTRODUCTION}

Wireless sensor networks (WSNs) comprise distributed autonomous sensors deployed to monitor environmental conditions such as pollution [1]. WSNs are significantly deployed due to the benefits of low costs [2], energy efficiency, and ease of scalable deployment. Sensors in WSNs commonly utilize the IEEE 802.11 technology. A typical WSN node is depicted in the block diagram in Figure 1 with four (4) basic units: the sensing, the processing and the storage, communication and the power. The sensing unit monitors the pollution level and acquires data that is processed by the microcontroller/microprocessors in the processing unit. The processed data is sent via the communication network to the main location where it is then analyzed and interpreted. The efficiency of the whole system described by the block diagram in Figure 1 is influenced by the throughput of the communication system and associated networking protocols. Some of the challenges that should be addressed for the communication system include selecting routing protocols; media access control, power management and coverage.

A typical sensor node that can be used in Figure 1 comprises a power source, transceiver module, air pollution monitoring payload and data storage entity. The sensor node acquires information on air data using its air pollutionmonitoring payload. The acquired data is pre-processed prior to being transmitted to appropriate entities via the sensor node's transceiver. The air pollution payload could comprise components such as smoke concentration, gas and dust particles sensing modules. 
Its primary responsibility would be to send its data to the moving node placed on buses and cabs driving on a predetermined route. Possible sensors that could be used to detect air pollution could include smoke concentration, gas and dust particles. Analyses would then be used to determine the extent of the pollution. There has been an increase in deaths due to air pollution because of the presence of volatile organic compounds in the air [2].

The concerns of determining the suitable WSN requires the design of a suitable wireless network to support the transfer of data acquired from stationary WSNs to mobile WSNs. Such a network plays an important role in the realizing the vision of the smart city concept. The network should have the capacity of enabling meaningful use of air pollution data. A meaningful use implies that the air pollution data should be utilized to enhance human health in smart cities. This is because an increase in deaths due to air pollution (via presence of volatile organic compounds) has been observed [2].

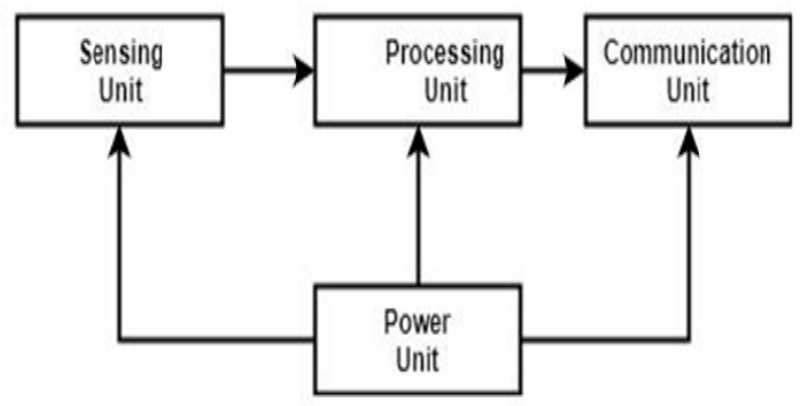

Figure 1: Block diagram WSN with four (4) Basic units

This paper proposes a WSN network architecture enabling the monitoring of air pollution. The proposed network also enables acquired data to be utilized in making decision about air quality and human health in smart cities. In the proposed network architecture, the sensor node acquires air pollution data and sends the acquired data to a server entity. The transmission of data to this server entity is realized via terrestrial wireless networks. In addition, the paper proposes the intelligent analysis of acquired air pollution data. The intelligent analysis is conducted to ensure power efficiency of the proposed WSN network. In addition, intelligent analysis of air pollution data enables a proactive determination of air pollution level via insights made from data analysis. This is done with the aim of ensuring that cities have acceptable and allowable air quality level suitable for healthy living.

The main contributions of the paper are in threefold. The first contribution is the design of a smart city and intelligent pollution monitoring system. The smart city incorporates a WSN network that enables the acquisition of air pollution data utilized in air quality analysis. In realizing the WSN network, the paper presents a novel energy efficient WSN network architecture that massively deploys sensor nodes for acquiring big data on air pollution in smart cities. The second contribution is the design of intelligent mechanisms enabling the analysis of acquired air pollution data via the sensors used in the proposed WSN network architecture. In this regard, the paper recognizes the suitable data and targets decision making from the perspective of different individual groups. Two individual groups are considered. These are individuals living in smart cities and individuals migrating to urban smart cities from rural areas. Thirdly, the paper presents an energy efficient sensor operational mechanism. This is done to ensure that massive deployment of sensor nodes in the proposed network architecture does not overwhelm the grid in the smart city i.e. the urban area.

The rest of this paper is organized as follows: Section II talks about the related work. Section III presents the theory behind the study in terms of the proposed method for pollution detection. Section IV discusses the preliminary results and discussion with explanation on the parameters to be used. Section V concludes this paper.

\section{RELATED WORK}

The amount of people moving from rural to urban areas is increasing and new cities will be needed to accommodate for this influx. The reason for this influx is mainly due to insufficient development in rural areas especially in developing nations. There are many challenges for urban living and addressing these challenges is important to 
ensure positive economic outcomes. This enables the realization of the vision of the smart city with relation to economic growth. Smart sites also attract investment which increases the overall well-being of the city and its citizens.

A smart city is an urban area that is advanced in terms of infrastructure, service delivery, communications and attractiveness. Information technology acts as the primary infrastructure and the medium for providing services to citizens in smart cities. Some of the benefits include increased efficiency and new technology adoption which most likely improves the quality of life of its citizens. Economic development and activity would be much more sustainable as these cities would attract young and intelligent professionals [3, 4].

This concept came during the economic crisis and coming up with alternative solution with cost reduction benefits is important to keep municipalities afloat. IBM began working on the smarter cities back in 2008 as part of its Smarter Planet Initiative. The idea spread like wild fire once companies and countries released its potential. Some of the countries that have researched and started implementation include China, UAE and South Korea. Cities that have already implemented such ideas include Aarhus, Cairo, Verona, Amsterdam and Vienna. The community needs to be actively involved in implementing a smart city for it to be a success. This includes energy saving as well as upgrading municipal infrastructure [4].

In [5], the authors carried out work on the Wireless Sensor Network (WSN) architecture for smart irrigation system. However, the work failed to address the issue of network congestion and power consumption. The authors in [6] present a new Wireless Sensor Network (WSN) architecture deployed to monitor air pollution. In this work i.e. [6], pollution data was analyzed to determine the optimal positions of sensors while reducing the node deployment cost. Furthermore, the discussion in [6], does not address the concern of sensor node power consumption. In addition, the authors in [7] propose a WSN used in a Smart Vehicle Monitoring system for Air pollution detection. The work carried out by authors in [7] achieves the monitoring of humidity temperature and other environmental factors.

Additionally, in [8], the authors carried out work on a prototype system with statistical data analysis based on Wireless Multi-sensor networks for smart cities. The discussions in [8] show that inconsistency, missing data and noisy data could be analyzed and extrapolation based on the measurements in time and space can be achieved. It is also demonstrated that short-term forecasts and smoothed maps estimation can be achieved via reliable estimates on the data consequently making the database to be represented geometrically. This can help the people to determine public services that may be needed.

Furthermore, in [9], an adaptive solution for smart cities using wireless sensor network for smart dumpster management was presented. The study utilizes the HC-SR04 ultrasonic sensors. The acquired data is used by servers operated by the municipal authority or provincial government server to determine the number of trucks and personnel for the collection of waste. Results presented in [9] suggest that planning and routing can be minimized via the time and conserves fuel consumed by the trucks. Finally, the proposed algorithm was used to enhance and optimize the fuel consumed by the trucks and time.

Besides this, in [10], the authors developed a scheme based on multiple mobile sinks in event-based wireless sensor networks using traffic conditions in smart city applications. In this study, results were obtained based on the computationally dynamic sinks movement in reactive sensor networks, which supports efficient adaptation to eventbased monitoring system in smart cities environment.

\section{POLLUTION DETECTION APPROACH - THEORY}

The discussion in this section presents the theory underlying the pollution detection approach. It is divided into two parts. The first part presents the proposed method for pollution detection. The second part focuses on the design and description of the proposed network architecture.

\section{A. Pollution Detection - Proposed Method}

The pollution detection process requires the use of a sensing unit at its core. The concerned sensing unit is designed and programmed to sense air pollutants [1]. The presence of air pollutants can be determined by monitoring parameters such as light level, temperature, humidity and pressure. The sensing unit also converts the sensed signals from an analog to a digital form. The sensor's processing unit i.e. the Microcontroller, process the signals sensed from sensor using onboard memory, operating system and associated circuitry. The sensor unit also has a transceiver that communicates with the router. The router receives information on sensed pollutant gas level from the sensor node. The sensed information is forwarded to a server entity. This server entity is known as the pollution server. The pollution server is sited in the administrator office or back office (central office). The sensor is powered 
is powered using one or two small batteries. There are also wireless sensors utilized in applications that use a fixed value, wired power source and do not use batteries as a power source.

Sensors hosting air pollution payload are deployed in clusters. A cluster comprises multiple sensors connected directly via a sink node. Each sensor communicates directly to the sink node eliminating latency [2]. In this work, the strategically placed clusters (radio components of deployed sensors) sited around the city are used for data collection. The aggregated data is transmitted to the mobile nodes traversing predetermined routes. The strategically placed clusters are realized as indoor units that are placed within short distance from the street. They also comprise weatherproof outdoor units placed along the predetermined route.

The best option for the mobile nodes would be to place them on the commercial buses or vehicles which move around the smart city. The Figure 2 shows nodes installed on the vehicles [3]. In the scenario presented in Figure 2 , the data acquired by deployed sensors is sent to strategically placed clusters i.e. radio components of deployed sensors. These clusters are sited at designated points in the smart city. Data received by the clusters are transmitted to mobile nodes deployed on vehicles traversing different parts in the smart city. The mobile nodes transmit this data to processing center realized via a cloud platform. This transmission occurs via data forwarding from the mobile nodes to the terrestrial wireless network.

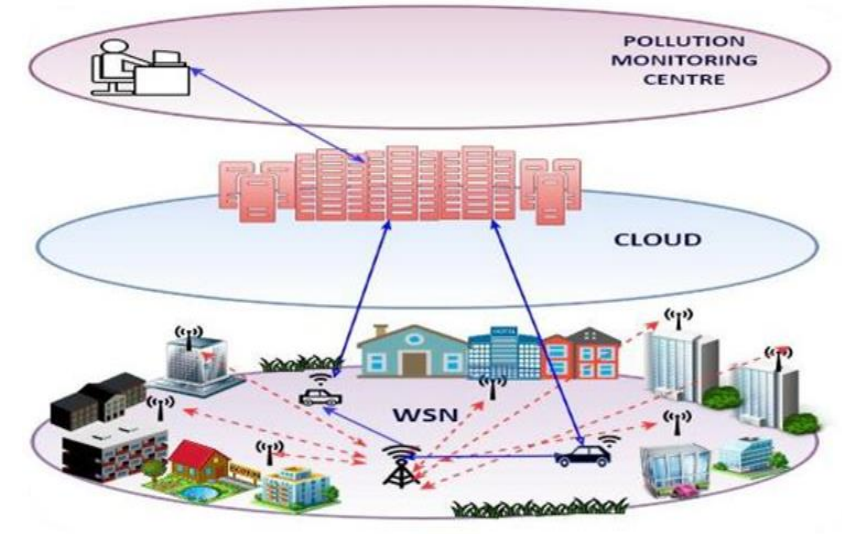

Figure 2. Vehicles and Smart cities collecting data from the stationary sensors around the city.

Example of data sample that would be collected could include smoke levels, dust and gas levels. The sink nodes i.e. radio nodes for deployed sensors search for neighbouring mobile nodes; a connection is established with the nearest mobile node.

The analysis of the data samples is done with the aim of identifying smart city locations with high concentrations of pollutants. This is done to enable the identification of locations where air pollution should receive focus. The analysis enables the source of the pollution to be estimated by means of statistical analysis of received data. The overall system would consist of many sub-systems scattered around the city. Each sub-system would have each of the different sensors to measure each type of pollution.

\section{B. Architecture and Network System}

In this section, the proposed architecture describing the connections between vehicles and the cloud platform is presented. The layout of the proposed architecture is shown in Figure 3. In Figure 3, the link between mobile nodes on vehicles and the cloud platform is realized via the LTE or 5G networks. This network choice is motivated by the need to achieve high throughput and low latency data transmission to enable quick decision making. The LTE technology is utilized on the vehicles which require a mobile connection.

Data transmission between massively deployed stationary nodes in the WSNs is realized via the ZigBee standard. The ZigBee standard has been chosen due to its low power consumption. The mobile nodes aboard the vehicles have ZigBee and LTE network communications capability. The stationary WSNs route the data to the moving vehicles. They will be ZigBee wireless sensors at the fixed locations around the route that transfer data to the 
moving buses. Once the data are collected by the vehicles, it will be uploaded using LTE/5G to a cloud network where it will be analyzed. The LTE / 5G is a new technology that is compatible with new devices and upgrades and the network scenario is shown in Figure 3.

Figure 4 below is a flowchart of the wireless sensor network that will be implemented in smart cities. The sensor nodes will collect data from the air and transfer it to the sink node using some communication methods. Once the sink nodes have collected data from all the nearby sensors, they will transmit the data to an online server via the mobile vehicles. The data will be processed as discussed earlier and can be viewed online [3].

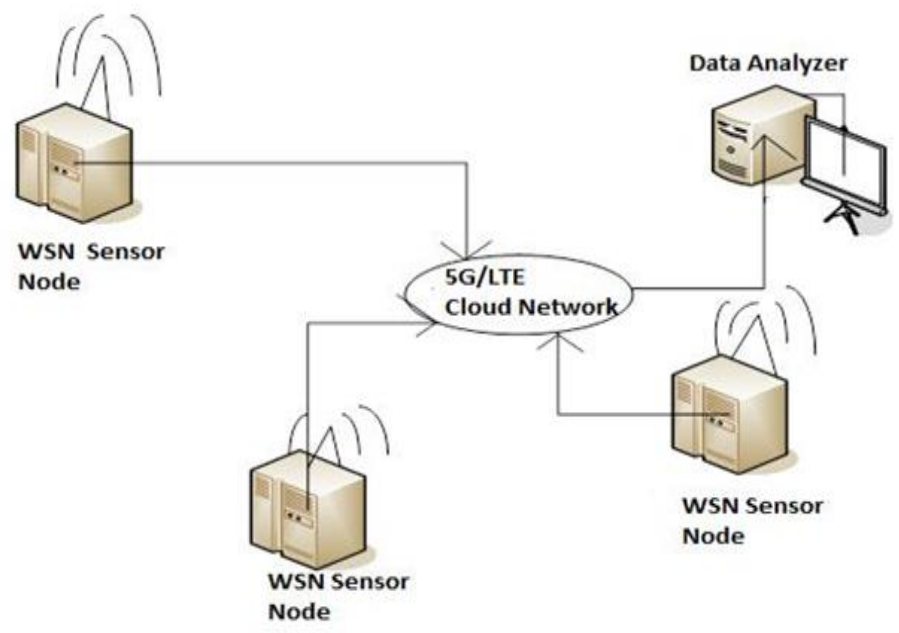

Figure 3. 5G/LTE network connecting the vehicles and smart cities to the cloud.

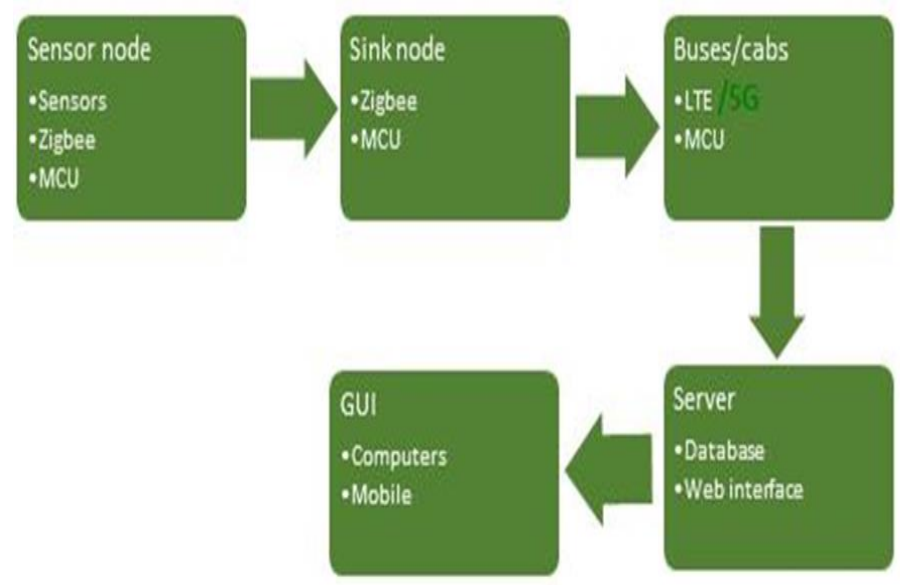

Figure 4. Adaptive wireless sensor network

\section{PRELIMINARY RESULTS AND DISCUSSION}

In this section, the preliminary results will be discussed based on Table 1 below. The Table 1 shows a different level of threshold that would be set abnitio for various monitoring environmental pollutants namely smoke, dust and dust. In this regard, there would be an indicator installed alongside the devices in the building and vehicles that keeps eye on the level of the pollutants in the vehicles and building in the smart city. As soon as it detects that there is a high or extreme level of pollutant, it quickly sends an alert which is connected to pollution monitoring center via 5G/ LTE where appropriate measures would be taken to control such occurrence. In this the proposed 
architecture, analyzed data can be viewed ubiquitously in the globe and can have access to the information about the pollution levels and rate through the $5 \mathrm{G} / \mathrm{LTE}$ cloud network system. In the full implementation of this proposed work in the nearest future, the pollution dataset of major cities in Nigeria such as Lagos, Kano and Abuja would be used. The values of simulation parameters employed are depicted in Table 3.

Table 1. Threshold Level \& Ranges for Different Pollutants.

\begin{tabular}{|l|l|l|l|}
\hline Pollutant & Middle & High & Extreme \\
\hline Smoke & $3-5$ & $6-7$ & $8-10$ \\
\hline Dust & $3-5$ & $6-7$ & $8-10$ \\
\hline Gas & $3-5$ & $6-7$ & $8-10$ \\
\hline Humidity & $3-5$ & $6-7$ & $8-10$ \\
\hline Oil & $3-5$ & $6-7$ & $8-10$ \\
\hline
\end{tabular}

Table 2. Simulation Parameters \& Values

Table 3: AQI parameters with pollutants.

\begin{tabular}{|l|l|l|l|l|}
\hline CO(ppm) & SO2 & NO2 & AQI & Category \\
\hline $0.00-4$ & $0.00-0.03$ & $* 1.98$ & $0-50$ & Good \\
\hline $4.01-9$ & $0.031-0.140$ & $* 1.98$ & $51-100$ & Moderately Good \\
\hline $9.01-13$ & $0.015-0.22$ & $* 1.98$ & $101-150$ & Unsuitable for Sensing equipment \\
\hline $13.01-16$ & $0.23-0.30$ & $* 1.98$ & $151-200$ & Unhealthy \\
\hline $16.01-27$ & $0.31-0.60$ & $0.649-1.237$ & $210-300$ & Very Unhealthy \\
\hline $40.40-49.9$ & $0.81-1.02$ & $1.602-2.034$ & $401-500$ & Extremely Hazardous \\
\hline
\end{tabular}

The performance analysis investigates the air quality index (AQI). The AQI is inferred from the index of the pollutant $I_{p}$ which is given as:

$$
I_{p}=\frac{\alpha_{H i}-\alpha_{L o}}{\beta_{H i}-\beta_{L o}} *\left(\gamma_{p}-\beta_{L o}\right)+I_{L o}
$$

Where:

Ip $=$ the index for pollutant $\mathrm{p}$;

$\gamma_{p}=$ the rounded concentration of pollutant $\mathrm{p}$.

$\beta_{H i}=$ the breakpoint that is greater than or equal to $\gamma_{p}$

$\beta_{L o}=$ the breakpoint that is less than or equal to $\gamma_{p}$.

$\beta_{H i}=$ the breakpoint that is greater than or equal to $\gamma_{p}$.

$\alpha_{H i}=$ the AQI value corresponding to $\beta_{H i}$.

$I_{L o}=$ the AQI value corresponding to $\beta_{L o}$.

The AQI obtained via simulation is evaluated for Lagos, Nigeria is presented in Table 3 and shown in Figure 5.

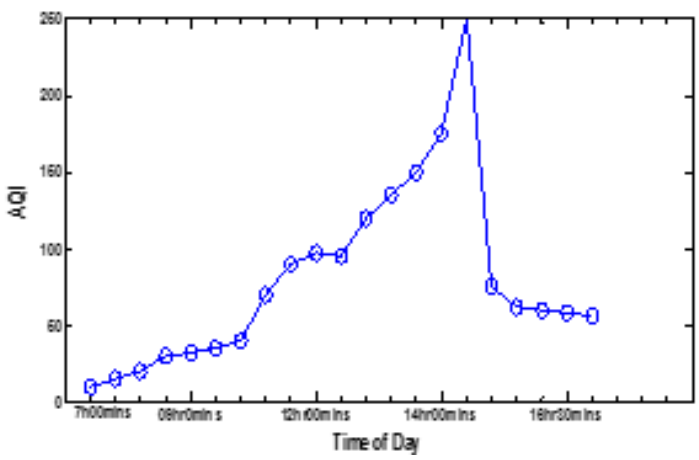

Figure 5: The result obtained for AQI 
Table 4: The AQI with time for the Investigated area (Oshodi-Isolo) in Lagos

\begin{tabular}{|l|l|l|}
\hline S/N & Time of Collection & Corresponding AQI \\
\hline 1 & $7: 00$ & 10 \\
\hline 2 & $7: 30$ & 15 \\
\hline 3 & $8: 00$ & 20 \\
\hline 4 & $8: 30$ & 30 \\
\hline 5 & $9: 00$ & 32 \\
\hline 6 & $9: 30$ & 35 \\
\hline 7 & $10: 00$ & 40 \\
\hline 8 & $10: 30$ & 70 \\
\hline 9 & $11: 00$ & 90 \\
\hline 10 & $12: 00$ & 97 \\
\hline 11 & $11: 30$ & 95 \\
\hline 12 & $12: 30$ & 120 \\
\hline 13 & $13: 00$ & 135 \\
\hline 14 & $13: 30$ & 150 \\
\hline 15 & $14: 00$ & 175 \\
\hline 16 & $14: 30$ & 250 \\
\hline 17 & $15: 00$ & 75 \\
\hline 18 & $15: 30$ & 62 \\
\hline 19 & $16: 00$ & 60 \\
\hline 20 & $16: 30$ & 58 \\
\hline 21 & $17: 00$ & 56 \\
\hline & & \\
\hline
\end{tabular}

\section{ACKNOWLEDGEMENTS}

The authors wish to thank the communications research group, University of Cape Town for financial support and the Department of Electrical Engineering at the University of Cape Town for their Lab and research tools support during this research project.

\section{REFERENCES}

[1] Goel, A., Ray, S., Agrawal, P., Chandra, N. Air Pollution Detection Based on Head Selection Clustering and Average Method from Wireless Sensor Network. Advanced Computing \& Communication Technologies (ACCT), Second International Conference, 2012, 434-438.

[2] Vijnatha, R.; Arvind; Kumar. Pollution Monitoring System using Wireless Sensor Network in Visakhapatnam. International Journal of Engineering Trends and Technology (IJETT), 2013, 4(4), 591-595.

[3] http://www.thehindu.com/features/homes-and-gardens/green-living/what-are-smartcities/article6321332.ece, (last accessed on 10 March 2018).

[4] Muhammad Saqib Jamil, Muhammad Atif Jamil, Anam Mazhar, Ahsan Ikram, Abdullah Ahmed, Usman Munawar. Smart Environment Monitoring System by Employing Wireless Sensor Networks on Vehicles for Pollution Free Smart Cities. Procedia Engineering, 2015, 107, 480-484.

[5] Trifun Savic; Milutin Radonjic. WSN Architecture for Smart Irri-gation System, 23rd International ScientificProfessional Conference on Information Technology (IT), 2018, 1-4.

[6] Ahmed Boubrima, Walid Bechkit; Herve Rivano. A New WSN Deployment Approach for Air Pollution Monitoring, 14th IEEE Annual Consumer Communications \& Networking Conference (CCNC), 2017, 455460.

[7] Suganya E.; Vijayashaarathi S. Smart Vehicle Monitoring System for Air Pollution Detection Using WSN, International Conference on Communication and Signal Processing, 2016, 0719-0722. 
[8] Balázs Csanád Csáji, Zsolt Kemény, Gianfranco Pedone, András Kuti, and József Váncza, Wireless MultiSensor Networks for Smart Cities: A Prototype System With Statistical Data Analysis, IEEE Sensors Journal, Vol. 17, No. 23, Dec. 1, 2017.

[9] Adian Fatchur Rochum and Riri Fitri Sari, Performance Comparison of IEEE 802.11n and IEEE 802.11ac, International Conf.on Computer,Control,Informatics \&its Application,2016. 